\title{
FINITE-TYPE INVARIANTS OF LEGENDRIAN KNOTS IN THE 3-SPACE: MASLOV INDEX AS AN ORDER 1 INVARIANT
}

\author{
VICTOR V. GORYUNOV and JONATHAN W. HILL \\ Department of Mathematical Sciences, Division of Pure Mathematics \\ The University of Liverpool \\ Liverpool L69 3BX, U.K. \\ E-mail: goryunov@liv.ac.uk, j.w.hill@liv.ac.uk
}

\begin{abstract}
We consider a contractible closure of the space of Legendrian knots in the standard contact 3-space. We show that in this context the space of finite-type complex-valued invariants of Legendrian knots is isomorphic to that of framed knots in $\mathbf{R}^{3}$ with an extra order 1 generator (Maslov index) added.
\end{abstract}

The idea to study invariants of generic objects via their degenerations goes back to Poincaré. It was very efficiently applied by Vassiliev in his work on knot invariants [10]. It turns out to be very fruitful in numerous other situations. For example, the study of invariants of Legendrian knots from a similar point of view was started by Arnold in $[1,2,3]$ where order 1 invariants of plane fronts and immersed curves were extensively investigated.

In $[8]$ the complete theory of finite type invariants of plane fronts without dangerous self-tangencies was constructed. The approach was analogous to that used in [6] for the study of invariants of regular plane curves. As in $[2,3]$, it was based on consideration of only immersed Legendrian curves. The latter causes certain inconvenience since the space of parametrisations of such curves has non-trivial topology. Therefore, to show, for example, that a certain codimension 1 stratum in the space of maps defines a co-cycle, one needs to check that its intersection with infinite order generators of the fundamental group vanishes. This task is sometimes rather tricky (see [3]). On the other hand, introduction of a contractible closure avoids such problems. But this can lead to appearance of some other finite-type invariants corresponding to the strata of the closure and to upgrading some order 0 invariants to positive order.

In the present paper we consider the closure of the space of immersed Legendrian

1991 Mathematics Subject Classification: Primary: 32S55. Secondary: 57M25.

The paper is in final form and no version of it will be published elsewhere. 
curves in the standard contact 3-space by allowing non-immersed curves tangent to the contact distribution only at their regular points. This results in a theory of finite-type invariants which treats the Maslov index as an invariant of order 1 instead of usual order 0 (cf. $[2,3]$ ). Similar to the traditional Vassiliev theory, we develop the corresponding diagrammatic theory for the Legendrian knot invariants of our setting. We show that the graded space of complex-valued invariants is isomorphic to that of framed knots in $\mathbf{R}^{3}$ $[6,7]$ with the Maslov index added as an extra generator.

The paper is organized as follows.

In Section 1 we recall some basic definitions.

In Section 2 we introduce the invariants we are studying and their extensions to Legendrian curves and fronts with mild singularities.

In Section 3 we construct the diagrammatic interpretation of finite-type invariants and formulate our main results: Theorem 3.4 on the homotopy between the fronts with mild singularities, which yields the reduction from the invariants to the diagrams, and Theorem 3.9 on the isomorphism between the graded spaces of the invariants and functions on the diagrams. The two theorems are proved in Sections 4 and 5 respectively.

1. Legendrian curves in the standard contact 3-space. The standard contact 3 -space is the space $J^{1}(\mathbf{R}, \mathbf{R})$ of 1 -jets of functions on the line with its canonical contact structure $\alpha=d u-y d x$, where $x$ is a point on the line, $u$ the value of a function and $y$ the value of the derivative. We fix the orientation $-\alpha \wedge d \alpha=d x \wedge d u \wedge d y$ of the jet space.

A Legendrian curve is a mapping $f: S^{1} \rightarrow J^{1}(\mathbf{R}, \mathbf{R})$ such that $f^{*}(\alpha) \equiv 0$. A Legendrian knot is an embedded Legendrian curve. A Legendrian knot type is a Legendrianisotopy class of Legendrian knots. All curves we are considering are oriented, that is, an orientation of $S^{1}$ is chosen.

It is very convenient to reduce the dimension and treat Legendrian curves via their projections to the $(x, u)$ - and $(x, y)$-planes.

The projections of generic Legendrian curves (any Legendrian knot type has such a generic representative) in the $(x, y)$-plane are curves which have only transversal double points as singularities: any contact plane in $J^{1}(\mathbf{R}, \mathbf{R})$ projects isomorphically to the $(x, y)$-plane. The forgotten coordinate $u$ can be restored by the integration of the 1 -form $y d x$ along the curve and hence the curves in the $(x, y)$-plane bound zero area. We call such curves zero-curves.

We fix the orientation $d x \wedge d y$ of the $(x, y)$-plane. The number $\mu$ of full turns made by the velocity vector of an immersed zero-curve while we trace the curve once (that is, its Whitney winding number) is called the Maslov index of the corresponding Legendrian knot. It is independent of the choice of a zero-curve representing the Legendrian knot.

The projections of Legendrian curves to the $(x, u)$-plane are called fronts. In generic case they have only transversal double points and semicubical cusps as singularities. Such a front has no vertical tangents: the $y$-coordinate of the knot in $J^{1}(\mathbf{R}, \mathbf{R})$ is $d u / d x$.

Cusps of a front one-to-one correspond to points of the zero-curve with vertical tangents, $d y / d x=\infty$. Therefore the Maslov index of a Legendrian knot is $\left(\mu^{+}-\mu^{-}\right) / 2$, where $\mu^{+}$and $\mu^{-}$are the numbers of positive and negative cusps (those oriented downwards 
and upwards respectively) of its front.

The main difference between our approach to the study of Legendrian knots and the traditional one (cf. $[2,3])$ is that we shall be very much basing on the consideration of non-immersed Legendrian curves. The most general way for them to appear is as follows.

Consider a generic local homotopy of a zero-curve contracting a loop. We integrate it to a homotopy of Legendrian curves and then project to a local homotopy of fronts (Figure 1).
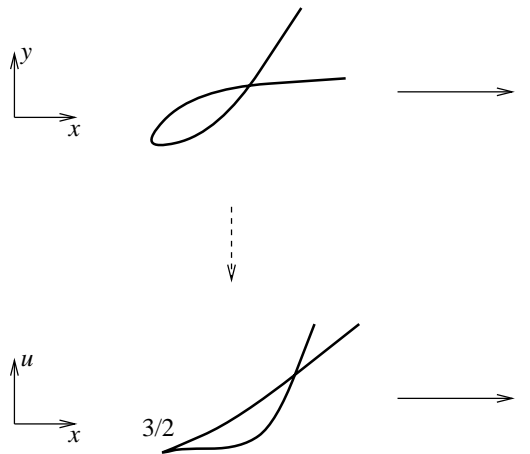
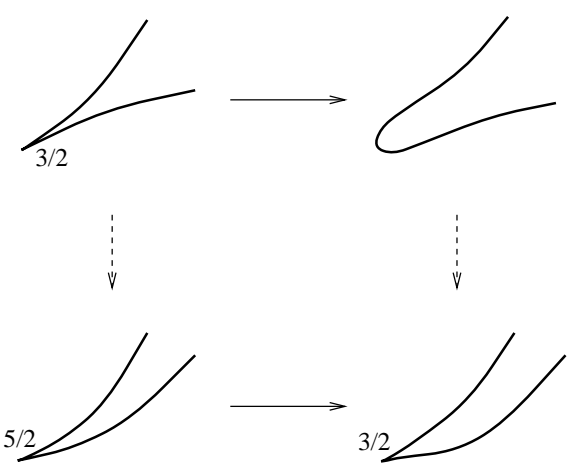

Figure 1. Contracting a loop on a zero-curve and the corresponding homotopy of fronts

The straightforward integration shows that at the bifurcation instant a front with a rhamphoid cusp (that is, a cusp of order 5/2) appears. The Legendrian curve which corresponds to it has a local parametrisation with the lowest terms $t \mapsto\left(t^{2}, t^{3}, t^{5}\right)$.

Similar to semicubical cusps, a rhamphoid cusp can be either positive or negative (the above definition should be just repeated). We split each of these types into two subtypes, left and right, depending on which side a cusp points to.

Another generic singularity of Legendrian curves, which is observed in their generic homotopies and will be crucial for our study, is a self-intersection (the curve is still immersed but fails to be embedded) which is best of all seen as a generic (that is, quadratic) self-tangency of the front.

\section{Invariants of Legendrian knots as invariants of their fronts}

2.1. The discriminant. We shall be working with Legendrian curves mainly in terms of their fronts.

Consider the space $\Omega$ of all maps of the form $p \circ f$, where $f: S^{1} \rightarrow J^{1}(\mathbf{R}, \mathbf{R})$ is an oriented Legendrian curve and $p: J^{1}(\mathbf{R}, \mathbf{R}) \rightarrow \mathbf{R}^{2}$ the standard projection from the jets to the $(x, u)$-plane. We call $\Omega$ the space of fronts.

Non-generic fronts form in $\Omega$ a hypersurface. It has six components which correspond to the possible degenerations in generic one-parameter families of fronts: concordant and discordant self-tangencies, triple points, cusp crossings, cusp births/deaths and rhamphoid cusps (Figure 2).

A self-tangency is concordant if the orientations of the tangent branches coincide and discordant if the orientations are opposite. 


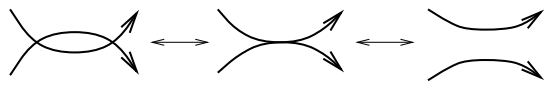

Concordant self-tangency bifurcation

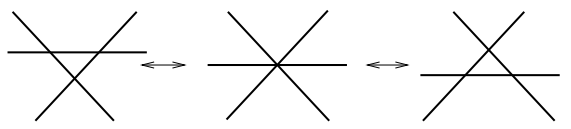

Triple point bifurcation

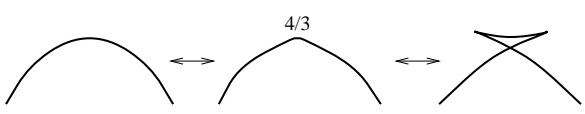

Cusp birth/death

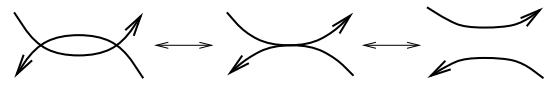

Discordant self-tangency bifurcation

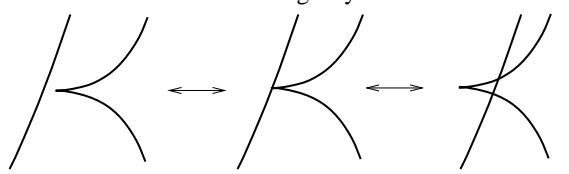

Cusp crossing bifurcation

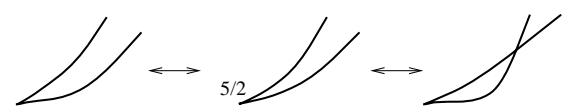

Rhamphoid cusp bifurcation

Figure 2. Degenerations in generic one-parameter families of fronts

Let the closures of the sets of fronts with concordant and discordant self-tangencies be $K_{C}$ and $K_{D}$ respectively and that of fronts with rhamphoid cusps be $R$. We consider the discriminant hypersurface $\Sigma=K_{C} \cup K_{D} \cup R \subset \Omega$.

DEFINITION 2.1. An invariant of fronts without self-tangencies and rhamphoid cusps is a function locally constant on $\Omega \backslash \Sigma$.

In what follows by 'invariants of fronts' we mean the invariants of this definition.

The space of such invariants coincides with the space of invariants of Legendrian knots in the 3 -space: lifting the homotopies of Figure 2 to the jet space one can easily see that only self-tangency and rhamphoid cusp bifurcations can change the corresponding Legendrian knots. Therefore, we shall make no difference between invariants of Legendrian knots and front invariants (of Definition 2.1).

We call a front which, in addition to the singularities of generic fronts, has a finite number of self-tangencies and rhamphoid cusps a singular front. A point on the smooth part of $\Sigma$ represents a singular front which has either one self-tangency or one rhamphoid cusp. A generic point on the $k$-tuple transversal self-intersection of $\Sigma$ represents a singular front with $n$ self-tangencies and $m$ rhamphoid cusps, $n+m=k$. In the latter case we say that a front has complexity $k$.

Now we co-orient $\Sigma$ : in the direction of increase of the number of double points of a front for the component $K_{C}$, and in the direction of decrease of the same number for both $K_{D}$ and $R$.
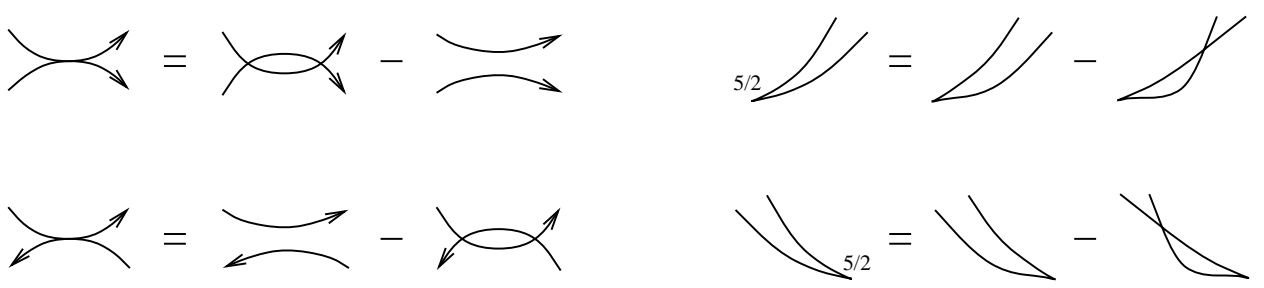

Figure 3. Extension of invariants to singular fronts 
As it is usually done in any theory of Vassiliev-type invariants, we extend invariants of fronts without self-tangencies and rhamphoid cusps to singular fronts via the recurrence relations of Figure 3. Here the fronts participating in each equation differ only by the shown fragments and the relations between the curves are in fact the relations between the values of an invariant on them. A rhamphoid cusp is distinguished by $5 / 2$ next to it. All these conventions will be used all through the paper.

Definition 2.2. An invariant is said to be of order at most $k$ if its extension to the set of singular fronts of complexity $k+1$ vanishes. An invariant is of finite type if it is of order at most $k$ for some $k<\infty$.

The way we define the extension implies that the Maslov index is an order 1 invariant: according to Figure 3 its value on a front with one rhamphoid cusp is \pm 1 .

2.2. Relations for extended invariants. A series of easy exercises on application of the rules of Figure 3 prove

Proposition 2.3. Any extended invariant of fronts satisfies the relations of Figure 4. In relation $(\mathrm{d})$, each of the fragments is a deformation of a triple point of self-tangency (Figure $5(\mathrm{~d})): \varepsilon_{i}=0$ if the destroyed self-tangency was concordant and $\varepsilon_{i}=1$ if it was discordant.

(a)

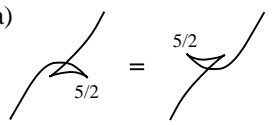

(b)

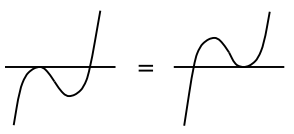

(c)

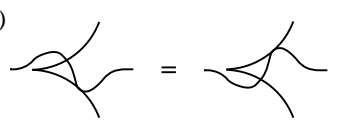

(d)

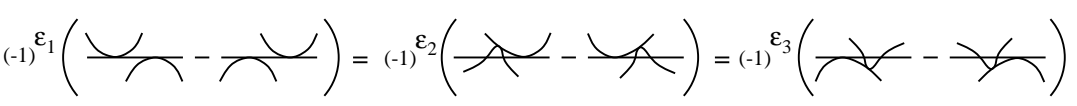

(e)

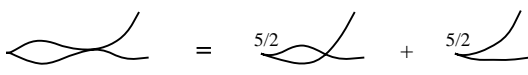

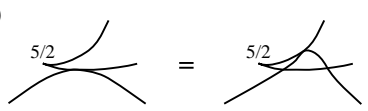

Figure 4. (a) Cuspidal commutativity, (b) Cubical two-term relation,

(c) Cuspidal two-term relation, (d) Four-term relation,

(e) Three-term relation, (f) Rhamphoid commutativity

Relations (b) and (d) may be found in [8]. In fact the four-term relation is nothing but the traditional $4 \mathrm{~T}$ relation of Vassiliev theory.

The first five relations come from the codimension 2 strata of the discriminant $\Sigma \subset \Omega$, the last one from codimension 3. All of them are defined and shown in Figure 5: all the fragments participating in relation $(\mathrm{x})$ of Figure 4 are deformations of the curve of Figure $5(\mathrm{x})$. For convenience, different branches are pictured in Figure 5 in different styles. 

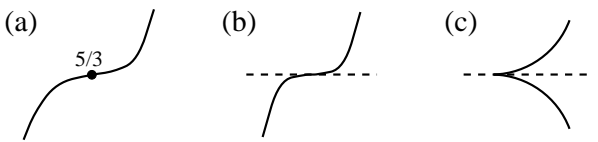

(d)

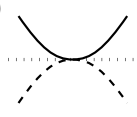

(e)

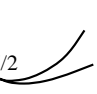

(f)

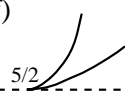

Figure 5. (a) Rhamphoid inflection, (b) Cubical self-tangency, (c) 3/2-cuspidal self-tangency, (d) Triple self-tangency point,

(e) 7/2-cusp, (f) Rhamphoid self-tangency

A more detailed explanation should be given to the nature of relation (a). It corresponds to the rotation of an ordinary cusp in the $(x, y)$-plane through the vertical direction. A curve of degree $5 / 3$ appears in the $(x, u)$-plane at the bifurcation instant (Figure 6).
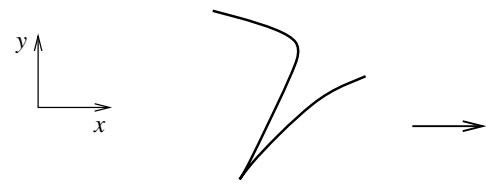

-
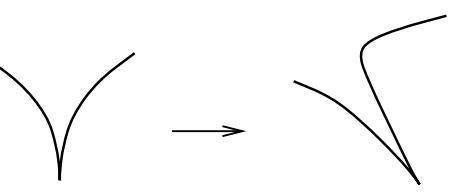

1
$\vdots$
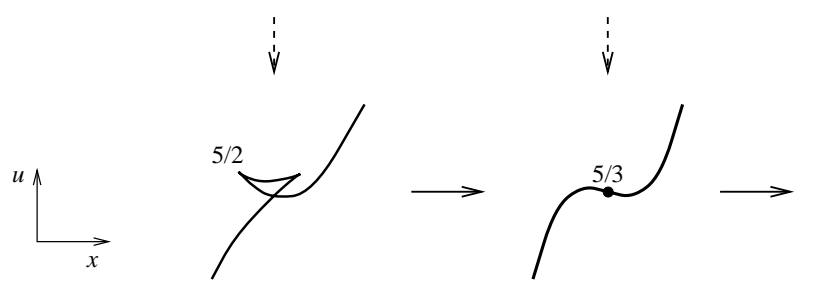

$\dot{v}$

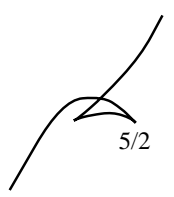

Figure 6. A rhamphoid inflection bifurcation

Remark 2.4. Adding time as a third coordinate to the $(x, u)$-plane and collecting all the fronts of the rhamphoid inflection bifurcation in a surface in the obtained 3-space, one gets the discriminant of the reflection group $\mathrm{H}_{3}$.

\section{Diagrams of fronts}

3.1. Chord diagrams with distinguished points. Consider an anti-clockwise oriented circle in the plane with a finite number of chords and distinguished points on it. All the endpoints of the chords and distinguished points are assumed to be distinct. We also assume that each of the distinguished points is equipped with a sign, either a plus or a minus.

DEFinition 3.1. A marked chord diagram is an equivalence class of the above objects up to orientation preserving diffeomorphisms of the circle. The complexity of a diagram is the number of its chords plus the number of its distinguished points.

To every singular front we associate such a diagram in a very straightforward way. Namely, we join by chords the pairs of points on the parametrising circle which are mapped to the self-tangencies of the front and the distinguished points are the preimages of the rhamphoid cusps. The sign of a distinguished point is the sign of the rhamphoid cusp. 
Definition 3.2. Two marked chord diagrams are similar if:

- Their unmarked versions (obtained by omitting all distinguished points and their markings from the diagram) coincide.

- The cyclic orderings of marked distinguished points on the two diagrams are the same.

3.2. Related fronts and enumeration of the strata. In knot theory two singular knots with the same chord diagram can be transformed one into the other by a homotopy (we call it generic) that stays almost all the time in the set of knots with this diagram and at a finite number of instants introduces an extra double point on a knot which immediately disappears. This provides enumeration of strata of singular knots which is crucial for Vassiliev theory. The analog of such enumeration in our case is as follows.

Consider the set $\Sigma_{k}$ of all fronts whose self-tangencies and rhamphoid cusps are subject to marked chord diagrams of complexity $k$ and which otherwise have only moderate singularities: transversal double points, semicubical cusps, 3/2-cusp crossings, cusp births (with a local normal form a parabola of degree $4 / 3$ ), third branches passing through selftangencies (transversally to the two tangent branches), rhamphoid cusp crossings (which are the obvious degenerations of the $3 / 2$-cusp crossings).

We are going to study generic homotopies of elements of $\Sigma_{k}$ which do not affect similarity classes of diagrams of their self-tangencies and rhamphoid cusps. These will be paths in $\Sigma_{k}$ enlarged with most of codimension 1 strata of its closure. The strata we are adding are:

$\Sigma_{k+1}$

$\Sigma_{k}^{\prime}$ : one of the self-tangencies of fronts from $\Sigma_{k}$ degenerates to either a cubical selftangency or a 3/2-cuspidal self-tangency;

$\Sigma_{k}^{\prime \prime}$ : one rhamphoid cusp and one self-tangency of fronts from $\Sigma_{k}$ join in a rhamphoid self-tangency;

$\Sigma_{k}^{\prime \prime \prime}$ : one rhamphoid cusp of fronts from $\Sigma_{k}$ degenerates to a rhamphoid inflection.

What we are not adding are degenerations of pairs of self-tangencies to triple selftangencies.

We set $\bar{\Sigma}_{k}=\Sigma_{k} \cup \Sigma_{k+1} \cup \Sigma_{k}^{\prime} \cup \Sigma_{k}^{\prime \prime} \cup \Sigma_{k}^{\prime \prime \prime}$.

Definition 3.3. Two fronts in $\Sigma_{k}$ are related if they can be joined by a generic homotopy in $\bar{\Sigma}_{k}$.

THEOREM 3.4. Two singular fronts are related if and only if their marked chord diagrams are similar.

Of course, related fronts are easily seen to have similar diagrams. The converse will be proved in Section 4 .

3.3. Symbols of finite order invariants. Let $R_{k}$ be the space of our front invariants of order at most $k$. An invariant of order $k$ is an element of $R_{k} \backslash R_{k-1}$.

Definition 3.5. The symbol of an order $k$ invariant is its restriction to the fronts of complexity $k$. 
Theorem 3.4 and relations (a), (b), (c), (f) of Figure 4 imply

COROllary 3.6. The value of the symbol of an order $k$ invariant on a front of complexity $k$ depends only on the similarity class of the marked chord diagram of the front.

For example, relation (a) of Figure 4 kills the difference between left and right rhamphoid cusps of the same sign.

Consider a symbol as a function on marked chord diagrams themselves rather than on their similarity classes. Then Proposition 2.3 translates into

COROLlaRY 3.7. The values of symbols of front invariants on marked chord diagrams satisfy the relations of Figure 7 .

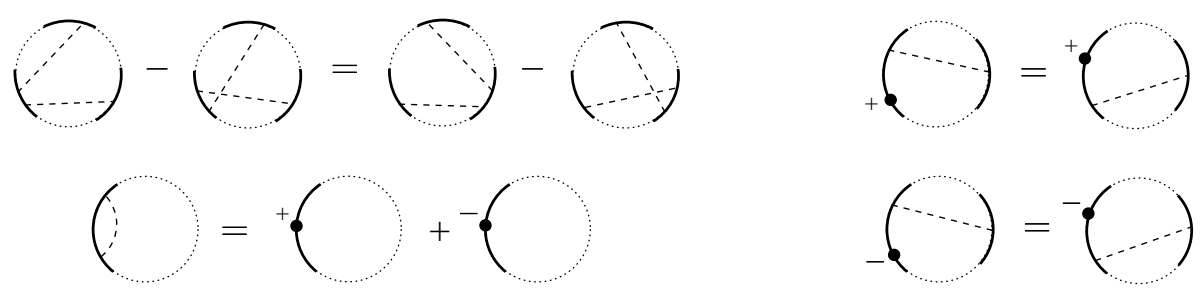

Figure 7. The four-term, three-term and floating point relations for symbols

Conventions of Figure 7 are similar to those used earlier for evaluating invariants on fronts: the relations on diagrams are actually relations on the values of a symbol on them, fragments of the diagrams based on the solid arcs are explicitly shown while those based on the dotted arcs are all omitted, diagrams participating in the same equation differ only by the fragments based on the solid arcs.

EXAmple 3.8. The symbol of the Maslov index vanishes on the 1-chord diagram. It is +1 on the diagram with one positive distinguished point and -1 on that with one negative point.

Let $\mathcal{M}$ be the space of all linear combinations of finitely many marked chord diagrams modulo the relations of Figure 7 (considered this time as relations between diagrams). It contains the commutative and cocommutative algebra $\mathcal{A}$ of ordinary chord diagrams modulo the four-term relation [9]. The multiplication in $\mathcal{A}$ is the connected summation of diagrams. From this one can easily check that the relations of Figure 7 guarantee that $\mathcal{M}$ itself is a commutative and cocommutative algebra with the connected summation of diagrams as a well-defined multiplication. Over char $\neq 2$, algebra $\mathcal{M}$ is isomorphic to the extension $\mathcal{A}[g]$ of $\mathcal{A}$ by an element

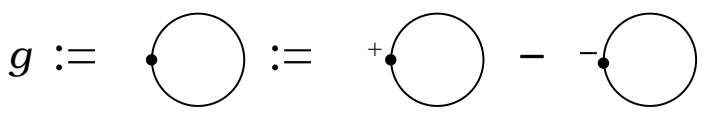

Again the multiplication is as above. Element $g$ is dual to $\mu / 2$.

Thus $\mathcal{M}$ can be treated over char $\neq 2$ as the space spanned by chord diagrams with distinguished (but unmarked) points considered modulo the four-term relation and 
unmarked floating point relation:

$$
(\cdots \cdots)=(\ldots-)
$$

Let the complexity $k$ part of $\mathcal{M} \simeq \mathcal{A}[g]$ be $\mathcal{A}[g]_{k}$ and $\mathcal{A}[g]_{k}^{*}$ its dual space. Corollary 3.7 implies that the symbol space $R_{k} / R_{k-1}$ is a subspace of $\mathcal{A}[g]_{k}^{*}$.

THEOREM 3.9. For complex-valued invariants $R_{k} / R_{k-1}=\mathcal{A}[g]_{k}^{*}$.

The proof of the equality involves a modification of the universal Vassiliev-Kontsevich invariant [9] and is given in Section 5 .

4. Normal forms of fronts: Proof of Theorem 3.4. We prove that all singular fronts with similar marked chord diagrams are related by bringing them to a common normal form. An example of what we are aiming at is given in Figure 8. Its main features are

- self-tangencies and rhamphoid cusps are gathered in different sections of the front separated by points $A$ (base point) and $B$;

- all self-tangencies are ordered (see Subsection 4.2) in a line and the connections between them are as simple as possible;

- all rhamphoid cusps form a sort of a staircase with the simplest connections between the steps.

We explain how to achieve each of the features in the corresponding subsections below.
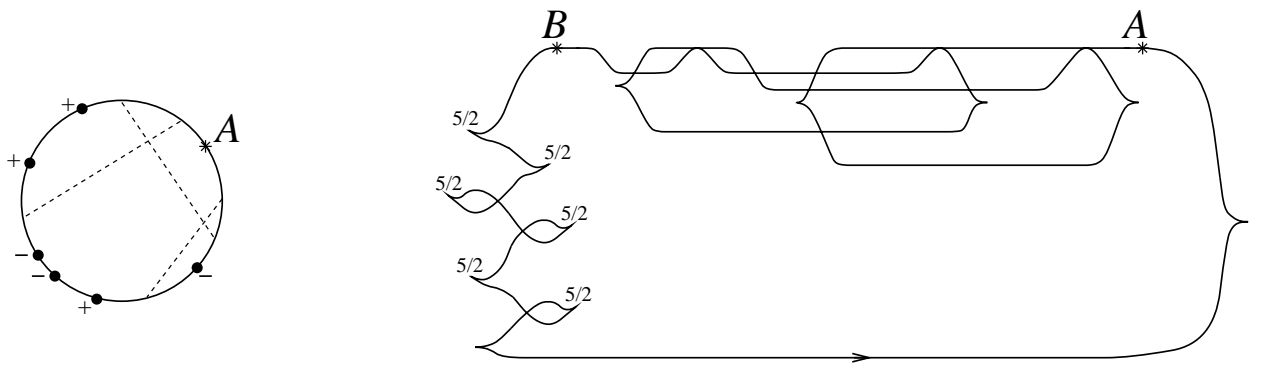

Figure 8. A front with a given marked chord diagram with a base point $A$ in the normal form

\subsection{Separating self-tangencies and rhamphoid cusps}

LEMMA 4.1. Any singular front is related to a singular front with the diagram in which distinguished points are not separated by endpoints of the chords.

Proof. Let us show that we can arrange free transportation of the rhamphoid cusps along a front through its self-tangencies and ordinary cusps in the direction (just to make it definite) of its orientation.

The major constraint in homotopies is as follows. Any point of a front can be moved vertically. But it may resist moving horizontally as our fronts do not have vertical tangents 
and local extrema of the horizontal coordinate $x$ on them can be achieved only at cusps, ordinary and rhamphoid.

We release a 5/2-cusp introducing a swallowtail next to it and arranging the floating cusp-pair of one rhamphoid cusp and one semicubical as in Figure 9.

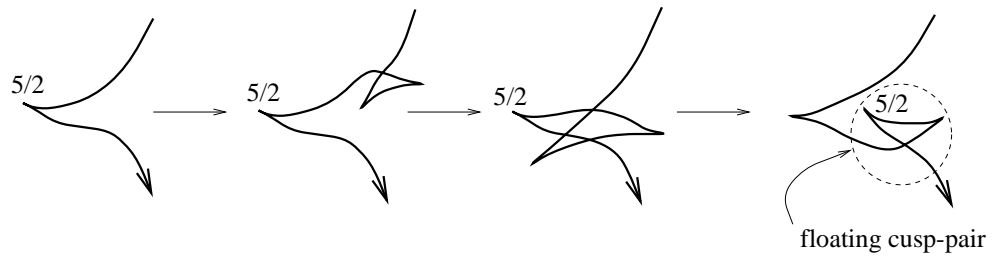

Figure 9. Achieving a floating cusp-pair

Moving such a pair through a self-tangency is obvious and causes no problems at all.

To pull the pair through an ordinary cusp, we must first ensure that its leading cusp (defined by the direction of the move, that is, by the orientation of the front, see Figure 10(a)) is semicubical. If it is not so, the swap can be done by the rhamphoid inflection bifurcation.

(a)

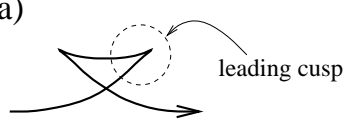

(b)

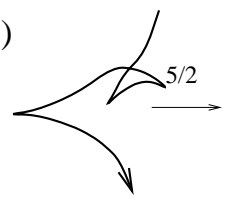

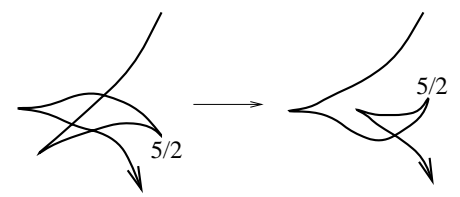

Figure 10. (a) The leading cusp in a floating cusp-pair, (b) Passing a floating cusp-pair through a semicubical cusp

Up to a rhamphoid cusp bifurcation we can assume that the sign of the cusp we would like to pass through coincides with that of the leading cusp in the pair. Now the passing itself can be done as shown in Figure 10(b).

Choosing a generic base point $A$ on a singular front, we collect all the rhamphoid cusps behind it (with respect to the orientation). Choosing now another generic point, $B$, just before the first $5 / 2$-cusp, we end up with two intervals: one, $A B$, containing all self-tangencies and the other, $B A$, containing all rhamphoid cusps (cf. Figure 8).

4.2. Simplification of the self-tangency part. Up to introduction of swallowtails we can assume that the front at both points $A$ and $B$ is oriented from the right to the left. To allow free horizontal moving in what follows we add a zig-zag (Figure 11) on either side of $A$ and $B$.

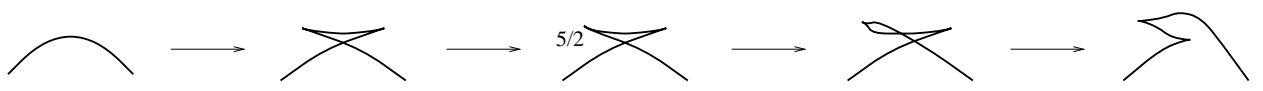

Figure 11. Zig-zag birth

Introducing swallowtails again and sliding self-tangencies along them, we ensure all the self-tangencies being concordant and, moreover, with all their local branches oriented from the right to the left. 
We order the branches of each self-tangency by the order of the visits to it starting from point $A$. The homotopy of Figure 12 allows us to assume that the branch of the second-time visit to each self-tangency is always below the branch of the first-time visit.

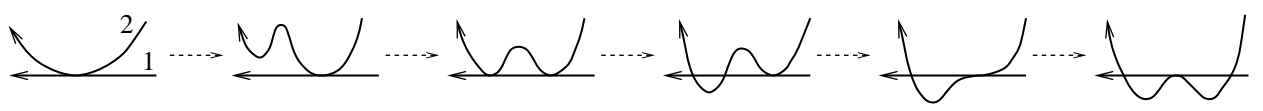

Figure 12. Arranging self-tangencies

We insert zig-zags around each point of self-tangency (Figure 13) to let it move freely along the plane.

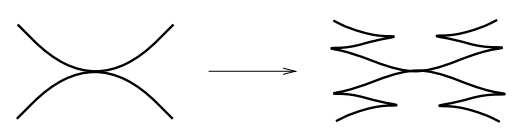

Figure 13. Releasing a self-tangency

Now we move points $A$ and $B$ to one $u$-level, $B$ to the left of $A$. We move all the self-tangencies to the same level, placing them from $A$ to $B$ in the order of the visits to their first-time branches if one starts from $A$ (cf. Figure 8).

To finish the normalisation of the self-tangency section of the front we have to simplify the connections between $A, B$ and self-tangency points. The procedure is based on

LEMMA 4.2. Let $S E$ be an interval of a generic front with regular starting and finishing points $S$ and $E, E$ to the left of $S$. Let the orientations at $S$ and $E$ be both right-to-left. There exists a homotopy of the interval which keeps its endpoints fixed, involves only generic bifurcations of Figure 2 and makes SE smooth.

Proof. Put all the interval from $S$ to $E$ into a box and scale the box down pulling out its connections with $S$ and $E$ (Figure $14($ a)).

(a)

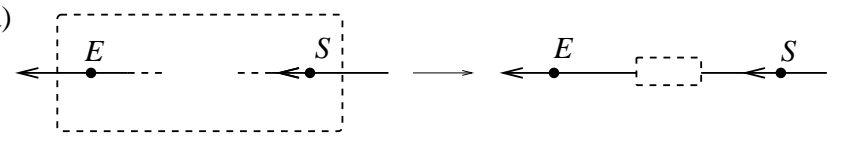

(b)

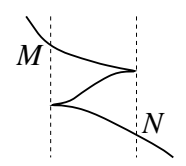

Figure 14. (a) Minimising the region, (b) The closest neighbouring cusps

Let us show that we can reduce any positive number of cusps in the box. We say that two cusps $c_{1}$ and $c_{2}$ of $S E$ are neighbouring if there are no other cusps between $c_{1}$ and $c_{2}$ on the front. Take the pair of neighbouring cusps with the minimal horizontal distance. Consider the strip of the plane bounded by the two vertical lines passing through these cusps. Let $M$ and $N$ be the points at which the fragment containing the cusps leaves the strip (Figure 14(b)). The interval $M N$ contains no other cusps. Moving the points vertically we bring $M N$ to the standard zig-zag or swallowtail shape and kill it by the standard homotopy. 
COROLLARY 4.3. Keeping all the conditions of Lemma 4.2, except for putting the starting point $S$ to the left of the finishing $E$, one can reduce the front interval to the form of Figure 15.

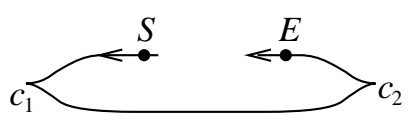

Figure 15. The simplest interval with the starting point to the left of the finishing

Proof. Up to rhamphoid cusp bifurcations we can assume that the first cusp $c_{1}$ after $S$ is positive and the last cusp $c_{2}$ before $E$ is negative. Moving the points vertically we make the intervals $S c_{1}$ and $c_{2} E$ monotonic. Now using the 'vertically reflected' version of Lemma 4.2 we smooth the interval between regular points just after $c_{1}$ and just before $c_{2}$ to obtain Figure 15.

Application of Lemma 4.2 and Corollary 4.3 finishes the normalisation of the selftangency section of our front.

4.3. Simplification of the part with rhamphoid cusps. Now we normalise the interval from $B$ to $A$. Up to creation of a floating cusp-pair (Figure 9) and applying a rhamphoid inflection bifurcation to it, we can assume that the first 5/2-cusp $r_{1}$ after $B$ points to the left. Similarly we ensure that the second rhamphoid cusp, $r_{2}$, points to the right, the third, $r_{3}$, to the left and so on in the alternating order.

We move the rhamphoid cusps along the plane so that $r_{1}$ is lower and to the left of $B, r_{2}$ is lower and to right of $r_{1}$ and so on in the descending and alternating order. Introducing a zig-zag just before $A$ (if needed) we align vertically $B$ with all the $r_{\text {even }}$ and separately align all the $r_{\text {odd }}$ (see Figure 8 ).

Applying Lemma 4.2 we smooth the intervals $B r_{1}, r_{1} r_{2}, r_{2} r_{3}, \ldots$. We can also arrange their intersections to be as minimal as possible so that only two half-branches of each negative rhamphoid cusp have exactly one point of transversal intersection (Figure 8).

Finally, if the number $m$ of $5 / 2$-cusps is even, we apply Corollary 4.3 to simplify the interval $r_{m} A$. If $m$ is odd, we take the last ordinary cusp $c_{\text {last }}$ before $A$ (the cusp can be made negative) and smooth the interval $r_{m} c_{\text {last }}$ applying Lemma 4.2.

This finishes the normalisation (see Figure 8). The final normal form is easily seen to be uniquely defined by the similarity class of the diagram of self-tangencies and rhamphoid cusps of a singular front once the base point $A$ is chosen on the parametrising circle. Here the uniqueness is that up to vertical moves of the smooth branches connecting the singularities.

\section{Modified Kontsevich integral}

5.1. The universal invariant. Similar to $[5,6,7]$ we define a version of the Kontsevich integral $[9,4]$ for Legendrian knots in $J^{1}(\mathbf{R}, \mathbf{R})$.

Represent the jet space with its standard coordinates $x, y, u$ as a product $\mathbf{C} \times \mathbf{R}$ with the complex coordinate $z=x+i u$ and real coordinate $y$.

We say that a Legendrian knot is Morse if $y$ is a Morse function on it (that is, all inflections of the front are non-degenerate). 
A Legendrian knot in the jet space is canonically framed by the negative $u$-direction (recall that the canonical Legendrian framing is that transversal to the contact structure).

Consider a non-singular oriented Morse Legendrian knot $\mathcal{L}$ and its small shift $\mathcal{L}_{\varepsilon}$ in the direction of the framing:

$$
(z, y) \mapsto(z, y)+\varepsilon(-i, 0)
$$

For all sufficiently small $\varepsilon>0, \mathcal{L}_{\varepsilon}$ is a knot which does not intersect $\mathcal{L}$. It inherits the orientation of $\mathcal{L}$.

The shift along the framing gives a one-to-one correspondence between points on $\mathcal{L}$ and $\mathcal{L}_{\varepsilon}$. For a point $\left(z^{\prime}, y\right) \in \mathcal{L}_{\varepsilon}$ this defines its ancestor $\left(z^{\prime \prime}, y\right) \in \mathcal{L}$ on the same $y$-level.

Let $y_{\min }$ and $y_{\max }$ be absolute extrema of function $y$ on $\mathcal{L}$. Take $k$ different non-critical values of $y: y_{\min }<y_{1}<y_{2}<\ldots<y_{k}<y_{\max }$. On each level $y=y_{j}$ we choose either one ordered pair of points $\left(z_{j}, z_{j}^{\prime}\right)=\left(z_{j}, z_{j}^{\prime}\right)\left(y_{j}\right) \in \mathcal{L} \times \mathcal{L}_{\varepsilon}$ or we distinguish one point $z_{j}^{\bullet}=z_{j}^{\bullet}\left(y_{j}\right)$ on $\mathcal{L}$.

Let $P$ be a set of pairs and single points obtained by such a choice. It defines the marked chord diagram of complexity $k$ as follows.

In each pair we substitute $z_{j}^{\prime} \in \mathcal{L}_{\varepsilon}$ by its ancestor $z_{j}^{\prime \prime} \in \mathcal{L}$. Knot $\mathcal{L}$ is the image of an immersion of a standard anti-clockwise oriented circle on the plane. If $z_{j} \neq z_{j}^{\prime \prime}$ we join the preimages of $z_{j}$ and $z_{j}^{\prime \prime}$ by a chord on the source circle. If $z_{j}=z_{j}^{\prime \prime}$ we draw a small chord between two arbitrary points on the circle which are very close to the preimage of $z_{j}$. Also we distinguish the preimages of the $m$ distinguished points $z_{j}^{\bullet}$.

We denote by $D(P)$ the equivalence class of the obtained chord diagram with distinguished points in the complexity $k$ part $\mathcal{A}[g]_{k}$ of the diagram algebra $\mathcal{A}[g]$ of Subsection 3.3 .

DEFINITION 5.1.

$$
\begin{aligned}
& \mathcal{Z}_{k}\left(\mathcal{L}, \mathcal{L}_{\varepsilon}\right) \\
& =\frac{1}{(2 \pi i)^{k}} \int_{y_{\min }<y_{1}<y_{2}<\ldots<y_{k}<y_{\max }} \sum_{P=\left\{\left(z_{j}, z_{j}^{\prime}\right)\left(y_{j}\right), z_{j}^{\bullet}\left(y_{j}\right)\right\}}(-1)^{\# P_{\downarrow}}\left(\bigwedge_{j=1}^{k} \omega_{j}\right) D(P) \in \mathcal{A}[g]_{k},
\end{aligned}
$$

where $P$ runs through all possible configurations of pairs and distinguished points on the levels $y_{j}$ of $\mathcal{L} \cup \mathcal{L}_{\varepsilon}, \# P_{\downarrow}$ is the number of points in the configuration $P$ at which function $y$ is decreasing along $\mathcal{L} \cup \mathcal{L}_{\varepsilon}$ and

$$
\omega_{j}= \begin{cases}\frac{d z_{j}-d z_{j}^{\prime}}{z_{j}-z_{j}^{\prime}} & \text { if there is a chord on the level } y_{j}, \\ i d \theta_{j} & \text { if there is a distinguished point on the level } y_{j},\end{cases}
$$

where $\theta_{j}$ is the angle from the positive $y$-direction to the velocity vector of the Legendrian knot at the point $\left(z_{j}^{\bullet}, y_{j}\right)$.

The angle in a contact plane is taken with respect to its standard orientation $d \alpha$.

DEFINITION 5.2.

$$
\mathcal{Z}_{k}(\mathcal{L})=\lim _{\varepsilon \rightarrow 0} \mathcal{Z}_{k}\left(\mathcal{L}, \mathcal{L}_{\varepsilon}\right), \quad \mathcal{Z}(\mathcal{L})=\sum_{k \geq 0} \mathcal{Z}_{k}(\mathcal{L}) \in \overline{\mathcal{A}[g]}=\prod_{k \geq 0} \mathcal{A}[g]_{k}
$$


Similar to [6] (cf. [9]) we have

THEOREM 5.3.

(i) The limit that defines $\mathcal{Z}_{k}(\mathcal{L})$ is finite.

(ii) $\mathcal{Z}_{k}(\mathcal{L})$ is invariant under homotopy in the class of Morse Legendrian knots in the jet space.

(iii) $\mathcal{Z}_{k}(\mathcal{L})$ is an invariant of order at most $k$.

Proof. All the claims are implied by the corresponding claims from $[6,7]$ (based on [9]) after the following observation.

Set as above

$$
\mathcal{Z}\left(\mathcal{L}, \mathcal{L}_{\varepsilon}\right)=\sum_{k \geq 0} \mathcal{Z}_{k}\left(\mathcal{L}, \mathcal{L}_{\varepsilon}\right) \in \overline{\mathcal{A}[g]}
$$

Consider a choice of the set $P$ of $n$ pairs and $m$ distinguished points on the Legendrian link $\mathcal{L} \cup \mathcal{L}_{\varepsilon}$. We fix the $n$ levels which contain pairs on the link. We vary the other $m$ levels between $y_{\min }$ and $y_{\text {max }}$ taking all possible allowed choices of $m$ distinguished points on them. Due to the floating point relation in $\mathcal{A}[g]$, no matter where we place the distinguished points the diagrams will all give the same element of $\mathcal{A}[g]$. In $\mathcal{A}[g]$ this element is the product of an $n$-chord diagram and an $m$-point diagram.

Thus

$$
\mathcal{Z}\left(\mathcal{L}, \mathcal{L}_{\varepsilon}\right)=\mathcal{Z}\left(\mathcal{L}, \mathcal{L}_{\varepsilon}\right)^{C} \times \mathcal{Z}\left(\mathcal{L}, \mathcal{L}_{\varepsilon}\right)^{D}
$$

where the factors are the sums of the integrals similar to the sum defining $\mathcal{Z}\left(\mathcal{L}, \mathcal{L}_{\varepsilon}\right)$ with the sets $P$ consisting purely of pairs and distinguished points respectively.

We see that $\lim _{\varepsilon \rightarrow 0} \mathcal{Z}\left(\mathcal{L}, \mathcal{L}_{\varepsilon}\right)^{C}$ is nothing but the Kontsevich invariant $\mathcal{Z}(\mathcal{L})^{f}$ from $[6]$ for the Morse framed knot $\mathcal{L}$ in $\mathbf{R}^{3}$ with its canonical Legendrian framing. All the claims of Theorem 5.3 are valid for all the fixed complexity components of it.

The second factor, $\mathcal{Z}\left(\mathcal{L}, \mathcal{L}_{\varepsilon}\right)^{D}$ does not depend on $\varepsilon$ at all and can be easily calculated explicitly:

$$
\mathcal{Z}\left(\mathcal{L}, \mathcal{L}_{\varepsilon}\right)^{D}=\exp (\mu g),
$$

where $\mu$ is the Maslov index of $\mathcal{L}$ and $g \in \mathcal{A}[g]$ is the one-point diagram. This follows from the fact that

$$
\frac{1}{2 \pi i} \int_{y_{\min }<y_{1}<y_{\max }} \sum_{P=\left\{z_{1}^{\bullet}\right\}}(-1)^{\# P_{\downarrow}} i d \theta\left(y_{1}\right)=\mu .
$$

Corollary 5.4. $\quad \mathcal{Z}(\mathcal{L})=\mathcal{Z}(\mathcal{L})^{f} \times \exp (\mu g)$.

As usual, the expression $\mathcal{Z}(\mathcal{L})$ is not invariant under deformations which change the number of critical points of the function $y$ on $\mathcal{L}$ and we have to adjust it to get a universal invariant.

As in [6], consider the non-Legendrian curve $U$ in the plane $\{\operatorname{Im} z=0\} \subset \mathbf{C} \times \mathbf{R}$ with the framing $(-i, 0)$ (Figure 16).

The series $\mathcal{Z}(U)^{f} \in \overline{\mathcal{A}} \subset \overline{\mathcal{A}[g]}$ is invertible since it starts with $1 \in \mathcal{A}[g]_{0}$. 


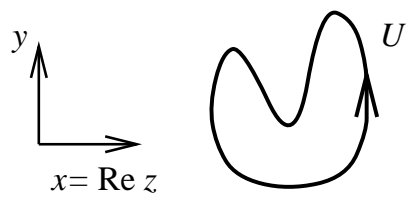

Figure 16. The curve $U$

Definition 5.5. The universal Vassiliev-Kontsevich invariant of a Morse Legendrian knot $\mathcal{L}$ in $J^{1}(\mathbf{R}, \mathbf{R})$ is

$$
\widetilde{\mathcal{Z}}(\mathcal{L})=\mathcal{Z}(\mathcal{L}) \times\left(\mathcal{Z}(U)^{f}\right)^{1-\frac{c}{2}} \in \overline{\mathcal{A}[g]},
$$

where $c$ is the number of critical points of the function $y$ on $\mathcal{L}$.

According to the last corollary

$$
\widetilde{\mathcal{Z}}(\mathcal{L})=\mathcal{Z}(\mathcal{L})^{f} \times \exp (\mu g) \times\left(\mathcal{Z}(U)^{f}\right)^{1-\frac{c}{2}}=\widetilde{\mathcal{Z}}(\mathcal{L})^{f} \times \exp (\mu g)
$$

where $\widetilde{\mathcal{Z}}(\mathcal{L})^{f}$ is the value of the universal invariant of framed knots in $\mathbf{R}^{3}$ on the knot $\mathcal{L}$ with its canonical Legendrian framing [6].

TheOREm 5.6 (cf. $[6,9])$. The series $\widetilde{\mathcal{Z}}(\mathcal{L})$ is an invariant of a Legendrian knot type.

Clearly, the complexity $k$ component $\widetilde{\mathcal{Z}}_{k}(\mathcal{L}) \in \mathcal{A}[g]_{k}$ of $\widetilde{\mathcal{Z}}(\mathcal{L})$ is an invariant of order at most $k$.

5.2. Proof of Theorem 3.9. Let $\mathcal{L} \subset J^{1}(\mathbf{R}, \mathbf{R})$ be a generic Legendrian knot and $\mathcal{F}$ its front. According to our earlier discussion, we identify invariants of Legendrian knots with invariants of fronts without self-tangencies and rhamphoid cusps. Therefore, we set the universal invariant $\widetilde{\mathcal{Z}}(\mathcal{F})$ of $\mathcal{F}$ to be the universal invariant $\widetilde{\mathcal{Z}}(\mathcal{L})$ of $\mathcal{L}$.

To prove the theorem, it is sufficient to show that any $n$-chord diagram $\mathcal{D} \in \mathcal{A}[g]$ with $m$ distinguished points is the lowest order term in a linear combination of the universal invariants of some generic fronts.

Let us order the distinguished points of $\mathcal{D}: p_{1}, \ldots, p_{m}$. Let $\mathcal{D}_{s_{1} \ldots s_{m}}, s_{j}=0,1$, be a marked chord diagram obtained from $\mathcal{D}$ by assigning the signs $(-)^{s_{j}}$ to the points $p_{j}$. According to the definition of the generator $g$ (Subsection 3.3) $\mathcal{D}=\sum_{s}(-1)^{s_{1}+\ldots+s_{m}} \mathcal{D}_{s_{1} \ldots s_{m}}$, with the summation taken over all the $2^{m}$ sequences $s=\left(s_{1}, \ldots, s_{m}\right)$.

Now take $2^{m}$ singular fronts $\mathcal{F}_{s_{1} \ldots s_{m}}$ with the marked chord diagrams $\mathcal{D}_{s_{1} \ldots s_{m}}$. We assume that these fronts coincide everywhere except small neighbourhoods of their rhamphoid cusps at which the difference is that standard one between the $5 / 2$-cusps of the proper signs. Then (cf. [9, 6] and Example 3.8)

$$
\sum_{s}(-1)^{s_{1}+\ldots+s_{m}} \widetilde{\mathcal{Z}}\left(\mathcal{F}_{s_{1} \ldots s_{m}}\right)=2^{n+m} \mathcal{D}+\text { higher order terms. }
$$

Remark 5.7. Similar to the approach of this paper one can study invariants of Legendrian knots in the standard solid torus $S T^{*} \mathbf{R}^{2}$ (isomorphic to $J^{1}\left(S^{1}, \mathbf{R}\right)$ ). In that case the graded space of finite type $\mathbf{C}$-valued invariants turns out to be isomorphic to the dual of a linear space spanned by chord diagrams with distinguished points which differs 
from $\mathcal{M} \simeq \mathcal{A}[g]$ by an additional marking: the base circle must be assigned an arbitrary integer $w$ (the Whitney winding number of a front), and each side of each chord must be assinged an integer (the Whitney numbers of the intervals into which the self-tangency cuts the front) so that the sum of the two numbers on each chord equals $w$. The relations are equipped with the appropriate markings as well. Cf. [5, 8].

\section{References}

[1] V. I. Arnol'd, Plane curves, their invariants, perestroikas and classifications, in: Singularities and Bifurcations, V. I. Arnold (ed.), Adv. Soviet Math. 21, Amer. Math. Soc., Providence, 1994, 33-91.

[2] V. I. Arnol'd, Topological Invariants of Plane Curves and Caustics, University Lecture Series 5, Amer. Math. Soc., Providence, 1994.

[3] V. I. Arnol'd, Invariants and perestroikas of plane fronts (in Russian), Trudy Mat. Inst. Steklov. 209 (1995), 14-64; English transl.: Proc. Steklov Inst. Mat. 209 (1995), 11-56.

[4] D. Bar-Natan, On the Vassiliev knot invariants, Topology 34 (1995), 423-472.

[5] V. V. Goryunov, Vassiliev type invariants in Arnold's $J^{+}$-theory of plane curves without direct self-tangencies, Topology 37 (1998), 603-620.

[6] V. V. Goryunov, Vassiliev invariants of knots in $\mathbf{R}^{3}$ and in a solid torus, in: Differential and Symplectic Topology of Knots and Curves, S. Tabachnikov (ed.), Amer. Math. Soc. Transl. Ser. 2, 190, Amer. Math. Soc., Providence, 1999, 37-59.

[7] V. V. Goryunov, Finite order invariants of framed knots in a solid torus and in Arnold's $J^{+}$-theory of plane curves, in: Geometry and Physics, J. E. Andersen, J. Dupont, H. Pedersen and A. Swann (eds.), Lecture Notes in Pure and Appl. Math. 184, Marcel Dekker, New York, 1997, 549-556.

[8] J. W. Hill, Vassiliev-type invariants of planar fronts without dangerous self-tangencies, C. R. Acad. Sci. Paris Sér. I Math. 324 (1997), 537-542.

[9] M. Kontsevich, Vassiliev's knot invariants, in: I. M. Gel'fand Seminar, S. Gel'fand, S. Gindikin (eds.), Adv. Soviet Math. 16, Part 2, Amer. Math. Soc., Providence, 1993, 137-150.

[10] V. A. Vassiliev, Cohomology of knot spaces, in: Theory of Singularities and its Applications, V. I. Arnol'd (ed.), Adv. Soviet Math. 1, Amer. Math. Soc., Providence, 1990, 23-69. 